\title{
Combining molecular and incomplete observational data to inform management of southern white rhinoceros (Ceratotherium simum simum)
}

\author{
Tarid Purisotayo $^{1,2}\left(\right.$ Nicholas N. Jonsson $^{1} \cdot$ Barbara K. Mable $^{1} \cdot$ Frederick J. Verreynne $^{3}$
}

Received: 5 November 2018 / Accepted: 6 March 2019 / Published online: 15 March 2019

(c) The Author(s) 2019

\begin{abstract}
Conservation efforts have preserved the southern white rhinoceros (SWR) in protected areas and have resulted in substantial overall growth in population size, but in small, fragmented populations in which inbreeding is an important risk. However, field observation of breeding often lacks sufficient accuracy to inform translocation strategies that are intended to increase genetic variation. The purpose of this study was to integrate microsatellite genotypes with an incomplete, field-observed pedigree to make inferences about mean kinship and basic demographic data that could be used to inform translocation programmes for SWR in a confined population in Botswana. Using this approach, we identified parents for 29 out of 45 offspring born in the reserve between 1993 and 2013 and detected eight non-breeding bulls with high mean kinship as candidates for translocation. The method also allowed inferences about demographic parameters that could influence the effectiveness of intervention strategies, such as age and timing of reproduction, and natal sex ratios. Importantly, the reproductive dominance of the bulls was not as skewed as expected after the original dominant bull was removed from the population, suggesting that closed populations can maintain multiple, simultaneously breeding males. The genetic data also confirmed that the accuracy of field-based parentage assignment was increased after implementation of an ear-notching programme. This study demonstrates the value of combining genetic information with ongoing surveillance to inform management of threatened populations, and of using mean kinship to inform metapopulation management by identifying candidates for translocation.
\end{abstract}

Keywords Kinship coefficient $\cdot$ Microsatellite $\cdot$ Parentage assignment $\cdot$ Pedigree $\cdot$ Translocation $\cdot$ White rhinoceros

\section{Introduction}

The southern white rhinoceros (Ceratotherium simum simum; SWR) was historically distributed over the land now designated as Botswana, Namibia, Swaziland, South Africa and Zimbabwe. The continental population was on the edge of extinction in the 1890 s, when approximately 50 to 100

Electronic supplementary material The online version of this article (https://doi.org/10.1007/s10592-019-01166-4) contains supplementary material, which is available to authorized users.

Tarid Purisotayo

tarid.p@msu.ac.th

1 Institute of Biodiversity, Animal Health and Comparative Medicine, University of Glasgow, Glasgow G12 8QQ, UK

2 Office of Academic Affairs, Faculty of Veterinary Sciences, Mahasarakham University, Maha Sarakham 44000, Thailand

3 Vet and Agric Consultants, Gaborone, Botswana individuals were all that remained in a single population at Hluhluwe-Umfolozi Park, Kwazulu-Natal, South Africa. Subsequent intensive protection efforts saw a rapid increase in the size of the population, which reached 1800 individuals by 1968 . With the application of newly developed protocols for translocation in the early 1960s (Player 1967), the population in Kwazulu-Natal became the founder of all African populations (Emslie and Brooks 1999). By 2015, the number of SWRs in Africa had reached 20,375 (Emslie et al. 2016).

In Botswana, the population of SWRs became extinct in the late nineteenth century as in all other countries within the species' former range. In 1967, four SWRs were relocated from Natal Park in South Africa into the Moremi Game Reserve in Okavango delta, and between 1974 and 1980, 91 more were relocated to Moremi and Chobe National Park (Tjibae 2001). However, the release areas, which are near several international frontiers and therefore subject to cross border poaching, were effectively unprotected and poaching almost wiped out the population. An intensive aerial survey 
of northern Botswana in September 1992 revealed only seven SWRs (leading to an estimate of a maximum of 10 animals in Chobe and 17 in Moremi); and between August and November of the same year, poachers were known to have taken another six SWRs (Department of Wildlife and National Parks of Botswana 2002). Subsequently, a new conservation strategy consisting of three phases of action was employed by the Department of Wildlife and National Parks of Botswana (DWNP). The main pillars of the new strategy were to: (a) protect SWRs in secure areas (confined reserves subject to close anti-poaching protection); (b) manage and monitor populations to achieve 5\% annual growth rates; and (c) re-introduce SWRs into large, unfenced national parks in the Okavango delta (Verreynne 2012). Initially, between 1994 and 1996, seven SWRs were captured at Chobe National Park and Moremi Game Reserve and were translocated into fenced sanctuaries elsewhere in Botswana (Tjibae 2001). Combined with further introductions from South Africa, this contributed to an increase in the number of SWRs in Botswana to 239 animals in 2015 (Emslie et al. 2016).

However, the population bottleneck of just over a century ago resulted in inbreeding and loss of genetic diversity, with consequent low number of alleles per locus of microsatellite markers (Florescu et al. 2003; Coutts 2009; Guerier et al. 2012). The mating system of the species, in which one dominant bull is expected to sire the majority of offspring in a population, results in low effective population size, exacerbating the problem of genetic drift, and generating many surplus bulls (Owen-Smith 1975). The International Union for Conservation of Nature (IUCN) suggested that translocation of SWRs is crucial to maintain levels of genetic diversity and it has become a routine management practice (Emslie et al. 2009). Translocation has been considered to be especially useful for the species because other means of enabling gene flow such as building habitat corridors are often not feasible, due to risk of poaching in unprotected areas. The population in this study is also located hundreds of kilometers away from the closest neighbouring reserves. Building corridors of such a distance as well as protecting SWRs in migration would be difficult and would demand a massive amount of resources. To be most effective, translocation requires a method for identifying the most appropriate individuals to be relocated, which is dependent on establishing a pedigree of relationships. However, an accurate field-observed pedigree can be difficult to obtain, particularly for wild animal species (Pemberton 2008). In the case of SWR, for example, calves may separate from their dams before they can be individually marked for later identification. The construction of pedigrees based on molecular markers has the potential to increase accuracy of parentage assignments to aid in the identification of candidates for translocations and to predict the inbreeding coefficient for all possible mating pairs. The kinship coefficient, also known as coancestry, of a given pair of individuals is the average probability that alleles at a locus randomly selected from those individuals are identical by descent (Ballou and Lacy 1995; Wang 2011). Alternatively, the kinship coefficient between a pair of individuals can be described as the expected inbreeding coefficient of their progeny. Individuals with high numbers of relatives generally show high mean kinship values (Mickelberg 2011); thus, young bulls with high mean kinship values would be expected to have a high risk of inbreeding in a population if they were retained and were to become dominant. Therefore, translocation of young bull with high mean kinship combined with regular removal of dominant bulls would help to prevent inbreeding within the population.

The purpose of this study was to integrate genotyping based on microsatellite markers with field observations to build a pedigree to allow inferences about mean kinship and basic demographic data of the population that could be used to inform translocation programmes for SWRs in a confined population in Botswana. The efficiency of permanent individual marking through ear-notching was also evaluated by determining the difference between observational assignment rates before and after the introduction of the approach.

\section{Materials and methods}

\section{Samples and population genetic parameters}

Due to security sensitivities and the risk of poaching, we are unable to explicitly provide the name and location of the studied reserve, which initially comprised a fenced area of approximately 4000 ha and has been expanded to 8600 ha since 2012. It is constantly patrolled by guards and protects a critically important population of about 55 SWRs. Between 1993 and 2013, 14 animals were introduced to the reserve and were considered to be founders, from 10 of which DNA samples were available from either blood samples or tissue samples retained after ear-notching for individual marking. The other four were either relocated or died before commencement of sampling; thus, no samples were available. Samples were collected during the routine procedures of individual marking or health checks from an additional 45/48 animals born in the park between 1993 and 2013. Three animals had died before they could be sampled. DNA was extracted using a phenol-chloroform protocol (Sambrook and Russell 2006), and genotyping was carried out in the Onderstepoort Veterinary Genetics Laboratory in South Africa, using 23 microsatellite loci, as previously reported (Harper et al. 2013).

To describe the genetic status of the population, we estimated population genetic parameters for 55 animals (10 founders and 45 offspring) in the population, including 
number of alleles per locus $\left(N_{a}\right)$, observed $\left(H_{o}\right)$ and expected $\left(H_{e}\right)$ heterozygosities, polymorphic information content $(P I C)$, frequency of null alleles $(F-n u l l)$ and the probability of non-exclusion for a candidate parental pair (NE-PP), using CERVUS 3.0.7 (Kalinowski et al. 2007). The NE-PP estimates the probability that a locus cannot exclude a randomly chosen parental pair within a population. Loci that deviated significantly from Hardy-Weinberg equilibrium (HWE) as tested using GENEPOP 4.2 (Raymond and Rousset 1995) were excluded. Inbreeding coefficient $\left(F_{i s}\right)$ and global deficit and excess of heterozygotes across loci were estimated to determine global deviation from HWE using Fisher's exact tests, as implemented in GENEPOP 4.2.

\section{Construction of population pedigree}

The workflow used in the construction of the pedigree for the study population is illustrated in Fig. 1. Briefly, the field observational records were used to construct a precursor pedigree (Pedigree A). The microsatellite genotypes were then incorporated to test whether the genetically possible parents from among the field-observed parents qualified (Pedigree B). In cases where the field-observed records and genotypes were unable to identify the parents for an offspring, maximum likelihood-based assignments were employed (Pedigree C). Bayesian-based parentage assignment was also implemented to confirm the results of Pedigree $\mathrm{C}$. The final pedigree (Pedigree D) was subsequently used to make inferences about mean kinship and to estimate demographic parameters.

\section{Using field observation to construct Pedigree A}

Between 1993 and 2013, the maximum population size in the reserve was 60 but the actual size at any given period was dynamic, being dependent on ongoing translocations. Field observations of mating, births and associations of females with calves were recorded by rangers and the veterinarian responsible for the reserve. The database of field observations included birth date and location of birth, introduction and relocation dates, and suggested a number of potential parents for each offspring. Parentage assignments for the offspring born in the reserve were constructed based on observed dam-offspring relationships. Dam was assigned to an offspring with high confidence when they were present together at the time of capture for individual marking. The level of confidence was reduced when an offspring was marked after it had separated from the dam. In such a case, the offspring was assigned to a set of possible dams. During the early period (1993-1995) following the establishment of the reserve, only one adult male and three adult females occupied the site. Although samples were not available for microsatellite genotyping, field-observed relationships between offspring and any of these founders were considered to have a high degree of confidence. To construct Pedigree A, unambiguous assignments were made if only one parent or one parental pair could be assigned to a given offspring.

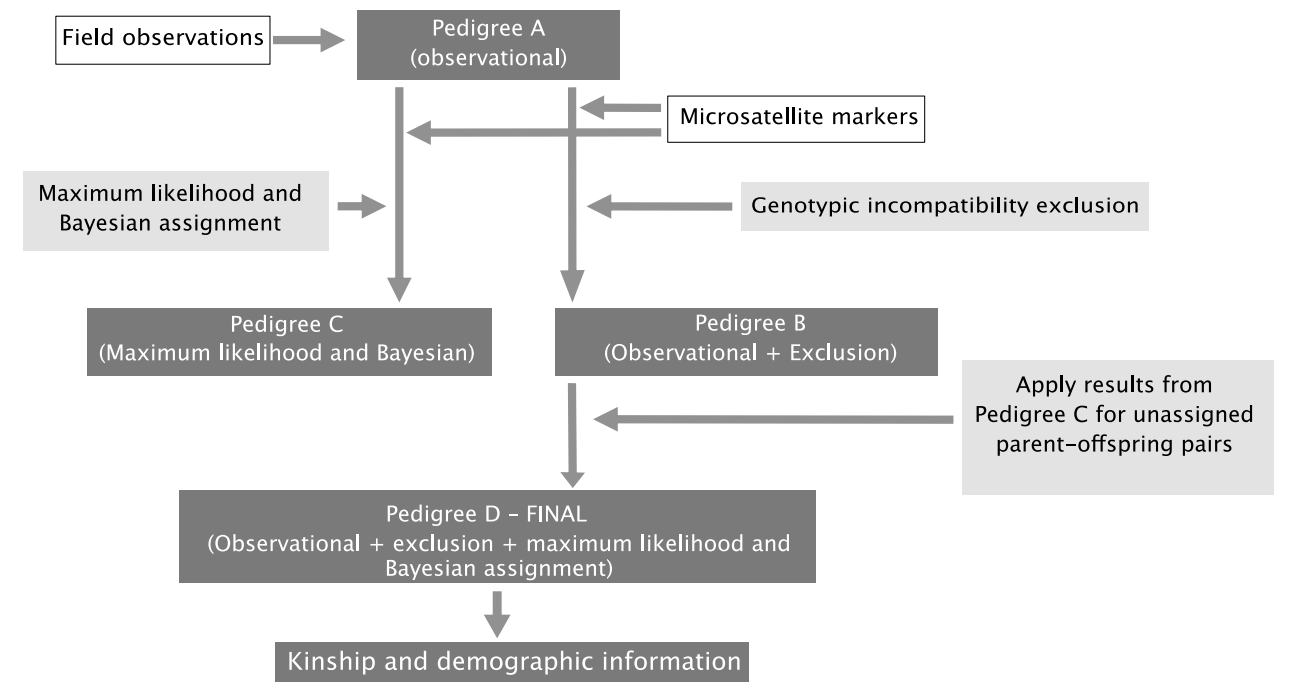

Fig. 1 Diagram of the workflow used in this study. Two types of inputs, field observations and microsatellite markers, are shown in white boxes; analytical processes and their outputs are presented in the light and dark grey boxes, respectively. Field observations were used to construct Pedigree A and genotypes were subsequently used to qualify the genetically possible parents (Pedigree B). Maximum likelihood-based and Bayesian-based assignments were incorporated (Pedigree C) in cases for which Pedigree B failed to unambiguously identify a parental pair for a particular offspring. The final pedigree based on the combination of all three approaches (Pedigree D) was used to make inferences about kinship and demographic information 
The efficiency of field-observed assignment was quantified as the proportion of unambiguous assignments divided by the total number of offspring. Offspring that were assigned to multiple sires or dams were left unassigned in this initial phase.

\section{Using observational and genotypic data to construct Pedigree B (exclusion)}

To narrow down possible parents for offspring who had been assigned multiple parents based on observations, genetically unlikely parents were excluded from the list of suggested parents of each offspring using $R$-package SOLOMON (Christie et al. 2013). Parents were excluded (considered to be impossible) whenever there was a mismatch between potential parents and offspring at one or more loci. A new, combined pedigree was then constructed (Pedigree B). The proportion of unambiguous assignments of Pedigree $A$ and Pedigree B was determined and compared. Any change in the proportion assigned would suggest the extent to which the microsatellite markers improved the observational pedigree.

\section{Using maximum likelihood and Bayesian approaches to construct Pedigree $\mathrm{C}$}

Prior to conducting parentage assignments using maximum likelihood and Bayes' theorem, information from field records was used to determine the theoretical reproductive window for each individual. The reproductive window defined the period during which an individual was at postpubertal age and was present at the site. This restricted the number of possible parents for both parentage assignment approaches to only those that were logistically possible. Six and five years of age were considered to be the ages of puberty for males and females, respectively (Rachlow et al. 1998). Sixteen months was used as the average gestation period (Rachlow and Berger 1998); a potential sire was excluded from the list of candidate parents for an offspring when it had been introduced to the reserve less than 16 months prior to the birth of the offspring, and an individual was excluded from the list if it had been removed from the reserve more than 16 months before.

We used CERVUS 3.0.7 (Kalinowski et al. 2007) to identify parents of 33 offspring whose possible parents did not include unsampled animals, as a high confidence level of assignment is achieved when all possible parents are sampled (Marshall et al. 1998). Likelihood-based assignment was conducted using the 18 polymorphic microsatellite loci that were in HWE. The LOD score between a parental pair and an offspring was interpreted as the natural logarithm of the likelihood ratio between the first and second hypothesis. The first hypothesis was that a tested trio comprised true parents and offspring, while the second hypothesis was that the trio was unrelated (Marshall et al. 1998). CERVUS then determined two types of delta scores: (1) trio delta, which was the difference between the LOD score of the most likely trio and the second most likely trio; and (2) critical delta obtained from computer simulation of parentage inference to identify the proper delta for the population in study. The simulation was conducted to obtain critical delta using the following parameters: 10,000 offspring with $90 \%$ of individuals in the population sampled; $98 \%$ of loci typed; allele frequencies of the population; and the confidence levels were set at $95 \%$ for strict critical delta. A trio was assigned unambiguously when the delta of the trio was greater than strict critical deltas that gave $95 \%$ confidence level.

To confirm the results obtained from likelihood-based parentage assignment and to allow estimation of parentage for duos, we used the Bayesian $R$-package SOLOMON (Christie et al. 2013) to assign parents for 76 parent-offspring pairs for which no unsampled founder was in the observational lists of possible parents (36 sire-offspring and 40 dam-offspring pairs). This package can incorporate prior probabilities based on genotype information alone. In this case, the prior probability was defined as the probability that at least one allele at each locus is shared between a randomly selected parent-offspring pair by chance. The prior probability was equal to the expected number of false parent-offspring pairs divided by the total number of possible parent-offspring pairs. A simulation was conducted to estimate the expected number of false pairs; here we set the number of simulations to 1000 and 50,000,000 for simulated data sets and genotypes, respectively (Christie et al. 2013). Parent-offspring pairs that had a prior probability equal to one were not further used to estimate the posterior probability. The posterior probability can be described as the probability that a possible parent-offspring pair is false given the allele frequencies of shared alleles. In this study, parent-offspring pairs that showed posterior probabilities of at most 0.05 were considered genuine relationships. If an offspring had multiple genuine relationships, the parent with the lowest posterior probability was considered a genuine parent. Pedigree $\mathrm{C}$ was then constructed using the results from likelihood-based assignments unless the results were contradicted by the Bayesian-based assignments, in which case the assignment was excluded.

\section{Using combination of pedigrees to construct the final Pedigree D}

The final pedigree was constructed using the combination of results obtained from Pedigree $\mathrm{B}$ and $\mathrm{C}$ to maximise the number of unambiguous assignments. Only in the situation that the Pedigree B failed to unambiguously assign a parent or a parental pair to an offspring, the result obtained from 
Pedigree $\mathrm{C}$ was implemented. In a case when more than one offspring born in the same year were assigned to the same mother, they all were considered to be unassignable.

\section{Efficiency of ear-notching to aid parentage assignment}

Ear-notching for individual identification and collection of samples for genotyping was introduced in 2006 and continuously conducted as newly born and introduced animals were later marked. The change in efficiency of observationalbased assignment after the introduction of ear-notching was tested. Twenty offspring observationally assigned to a mother whose genotype was available were used to compare the difference between assignment rates of: (1) offspring born before ear notching was implemented (1993-2008; $n=7$ ); and (2) offspring born after this change in management practice (2009-2013; $n=13)$, using Fisher's exact tests (Agresti 1992). The implementation of ear-notching had started in 2006, but 2008 was used as a cutoff to ensure that a substantial proportion of animals were marked, and the benefit of the approach could be detected.

\section{Demographic parameters}

Pedigree D was subsequently used to estimate population demographic parameters, including: (1) annual calving rate $(A C R)$, defined as the annual percentage of dams that gave birth of the total number of reproductive age dams; (2) percentage of herd growth ( $H G$-see Eq. 1, below), described as net increase in size of the herd as a result of newborn calves divided by the size of the herd at the beginning of the year (Ververs et al. 2017); (3) mean age of first calving of dams born in the reserve; (4) mean total number of offspring produced per sire and dam over the period of observation; (5) natal sex ratio; (6) the effective population $\left(N_{e}\right)$; and (7) calving interval $(C I)$ for multiparous females. The estimation of percentage of $H G$ shown in Eq. 1 accounted for only the effect of newborn SWRs to the annual population growth, with 6 and 5 years old considered adult ages for males and females, respectively (Rachlow et al. 1998).
50:50 males to females, and skewness was evaluated using $G$-test statistics (Kretzschmar 2001). The effective population size $\left(N_{e}\right)$ is here described as the number of breeding individuals (Falconer 1960) and was estimated by taking the sum of the number of individuals that contributed to offspring born in the reserve. For parameters that required the birthdate for estimation we used the first of January or the first of a given month in cases where the field observations for an individual recorded only a year or month of birth, respectively.

\section{Pedigree plot, kinship coefficients, mean kinship}

We used the $R$-package PEDANTICS (Morrissey and Wilson 2010) to draw Pedigree D. The $R$-package Kinship2 (Sinnwell et al. 2014) was then used to estimate all pairwise kinships based on the assumption that all founder animals were unrelated. The elements in kinship matrices showed pairwise kinships between individuals that were computed by identifying the probabilities that alleles randomly drawn from a pair of individuals are identical by descent. Mean kinship of an individual was estimated by averaging of all pairwise kinship between the individual and other individuals within the population, including itself (Ballou and Lacy 1995). Bulls with no evidence of contributing paternity, whose individual mean kinship was higher than the population mean kinship, were considered the best candidates for translocation.

\section{Results}

\section{Population summary statistics}

Three out of 23 loci were found to be monomorphic (DB23, IR22, and SR74). For the 20 polymorphic microsatellite loci genotyped, three deviated from HWE - two of them highly significantly (DB66, IR12; p-value $<0.01$ ) —and so were excluded from the estimation of means of population genetic parameters. Note that locus IR22 was found to be monomorphic in this population but was reported to be

$\% H G=\frac{\text { no. of calves born }- \text { no. of deaths }}{\text { no. of resident adults }+ \text { no. of adults arriving that year }} \times 100$

The age of first calving for each of the dams born in the reserve was determined by estimating the interval between its birthdate and the date of its first calving. The duration between two consecutive calvings was used to determine $C I$ values. Bulls were considered to be dominant when they sired more than $50 \%$ of all the offspring born in a given year. Natal sex ratio was estimated based on an expectation of polymorphic elsewhere (Scott 2008). Based on the final set of 18 loci, means of population genetic parameters did not indicate high levels of inbreeding: $H_{o}$ and $H_{e}$ were 0.426 and 0.409 , respectively; $P I C=0.340$; and $F_{i s}=-0.0406$ (Table 1). Even though the most variable locus only had four alleles (compared to an overall mean $N_{a}$ of 2.50 across loci), there was a high potential to exclude parents based on 
Table 1 Population summary statistics of 55 animals in the reserve in computed by CERVUS 3.0.7 and GENEPOP 4.2

\begin{tabular}{llllllllrr}
\hline Locus & $N_{a}$ & $N$ & $H_{o}$ & $H_{e}$ & $P I C$ & $N E-P P$ & $H W E$ & $F_{i s}$ & $F($ null $)$ \\
\hline 32A & 3 & 55 & 0.564 & 0.558 & 0.455 & 0.614 & NS & -0.0109 & -0.0075 \\
DB44 & 3 & 55 & 0.309 & 0.316 & 0.290 & 0.724 & NS & 0.0229 & 0.0471 \\
7B & 3 & 55 & 0.545 & 0.492 & 0.433 & 0.605 & $p=0.04$ & -0.1088 & -0.0328 \\
7C & 3 & 54 & 0.704 & 0.578 & 0.509 & 0.540 & NS & -0.2202 & -0.0966 \\
BlRh1B & 2 & 55 & 0.418 & 0.481 & 0.363 & 0.726 & NS & 0.1309 & 0.0649 \\
DB66 & 4 & 55 & 0.182 & 0.428 & 0.375 & 0.659 & $p<0.01$ & 0.5776 & 0.3908 \\
DB52 & 3 & 55 & 0.636 & 0.615 & 0.531 & 0.531 & NS & -0.0350 & -0.0320 \\
BR6 & 2 & 55 & 0.400 & 0.400 & 0.318 & 0.753 & NS & 0.0008 & -0.0041 \\
DB1 & 2 & 55 & 0.273 & 0.238 & 0.208 & 0.824 & NS & -0.1489 & -0.0692 \\
BIRh1C & 2 & 55 & 0.400 & 0.416 & 0.327 & 0.748 & NS & 0.0396 & 0.0155 \\
12F & 2 & 48 & 0.521 & 0.495 & 0.370 & 0.722 & NS & -0.0538 & -0.0311 \\
B1Rh37D & 2 & 55 & 0.018 & 0.018 & 0.018 & 0.982 & ND & 0.0000 & -0.0008 \\
32F & 3 & 55 & 0.327 & 0.282 & 0.249 & 0.780 & NS & -0.1641 & -0.0847 \\
SR63 & 2 & 55 & 0.509 & 0.476 & 0.361 & 0.728 & NS & -0.0693 & -0.0377 \\
IR10 & 2 & 55 & 0.291 & 0.323 & 0.269 & 0.783 & NS & 0.1000 & 0.0476 \\
IR12 & 2 & 55 & 0.218 & 0.364 & 0.296 & 0.767 & $p<0.01$ & 0.4033 & 0.2466 \\
SR262 & 2 & 55 & 0.382 & 0.409 & 0.323 & 0.750 & NS & 0.0659 & 0.0292 \\
SR268 & 3 & 54 & 0.259 & 0.251 & 0.234 & 0.773 & NS & -0.0327 & -0.0078 \\
SR281 & 3 & 55 & 0.655 & 0.638 & 0.561 & 0.503 & NS & -0.0256 & -0.0133 \\
RH12 & 2 & 52 & 0.462 & 0.379 & 0.305 & 0.761 & NS & -0.2216 & -0.1034 \\
Mean & 2.5 & 54.4 & $0.426^{\mathrm{a}}$ & $0.409^{\mathrm{a}}$ & $0.340^{\mathrm{a}}$ & $0.0018^{\mathrm{a}, \mathrm{b}}$ & $p<0.01^{\mathrm{a}, \mathrm{c}}$ & $-0.0406^{\mathrm{a}}$ & 0.0160 \\
\hline & & & & & & & & & \\
\end{tabular}

$N_{a}$ : number of alleles at a locus; $N$ : number of typed individuals at the locus; $H_{o}$ : observed heterozygosity; $H_{e}$ : expected heterozygosity; $P I C$ : polymorphic information content; $N E-P P$ : non-exclusion probability of a parental pair at the locus; $H W E$ : significance of deviation from HWE, $N S$ : not significant, $N D$ : not done, $p$ : statistic $p$-value; $F_{i s}$ : inbreeding coefficient; $F$-null: frequency of null alleles

${ }^{a}$ Highly HWE-deviated loci (DB66 and IR12) were removed from mean estimation

${ }^{\mathrm{b}}$ Combined probability of $N E-P P$

${ }^{\mathrm{c}}$ Global heterozygote deficit $(p$-value $=0.990)$ and excess $(p$-value $=0.009)$ the low combined probability of $N E-P P$ at 0.0018 . Global tests for heterozygote deficit and excess across the 18 loci revealed that the population contained significantly high heterozygosity ( $p$-value $<0.01)$.

\section{Efficiency of parentage assignments using the combination of approaches and the usefulness of ear-notching for parentage assignments}

The proportions of unambious assignments for distinct parentage assignment approaches are provided in Table 2 and the assignments made for all offspring are provided in the Supplementary Table 1. Using field observation alone: five offspring could be assigned to a parental pair; one and 20 offspring were assigned to a sire or dam, respectively. The application of the exclusion approach (Pedigree B) increased the number of assignable offspring: 12 offspring were assigned to a parental pair; 10 and nine offspring were assigned to a sire and dam, respectively. Using the likelihood-based parentage assignment approach, we could identify a parental pair for 23 out of 33 offspring. Only five out of 76 possible parent-offspring pairs were considered genuine
Table 2 The proportion of unambiguous assignments for trios, sire-, and dam-offspring duos for each approach

\begin{tabular}{llll}
\hline Approaches & \multicolumn{3}{l}{ Proportion of unambiguous assignments } \\
\cline { 2 - 4 } & Trio & Sire & Dam \\
\hline Field-observed & $5 / 45$ & $1 / 45$ & $20 / 45$ \\
Field-observed plus & $12 / 45$ & $10 / 45$ & $9 / 45$ \\
$\quad$ exclusion & & & \\
Likelihood & $23^{\mathrm{a}} / 33^{\mathrm{b}}$ & - & - \\
Bayesian & - & $4 / 36^{\mathrm{c}}$ & $1 / 40^{\mathrm{c}}$ \\
Combination & $29 / 45$ & $6 / 45$ & $4 / 45$ \\
\hline
\end{tabular}

${ }^{\text {a }}$ The assignments that exceeded the strict critical delta were considered unambiguous

${ }^{\mathrm{b}}$ Likelihood assignments were conducted for 33 offspring for which genotypes of all possible sires and dams were available

${ }^{\mathrm{c}}$ Bayesian assignments were conducted for offspring for which genotypes of all possible sires and dams were available but excluding duos involving unsampled founders

using Bayesian-based assignment, three of which were consistent with the likelihood-based assignment. One was inconsistent (ID 172) but the parent with the second lowest 
posterior probability (not statistically significant) was the same mother that was assigned using the likelihood-based assignment. The other inconsistent assignment was made for an offspring that was not examined using CERVUS but the assigned parent was identical to the parent suggested by Pedigree B (ID 121). The final pedigree of 45 offspring revealed 29 assignable trios, six sire-offspring duos, and four dam-offspring duos. After the introduction of ear-notching, the assignment rate of observational dam-offspring relationships was significantly improved ( $p$-value $=0.02$ ), with only $1 / 7$ compatible relationships prior to 2008 and 10/13 after 2008 .

\section{Using mean kinship to identify individuals for translocation}

Pedigree D, drawn using PEDANTICS, is shown in Fig. 2. A population mean kinship of 0.0483 was estimated and all pairwise kinship coefficients are shown in Supplementary Table 2. Unassignable offspring were given no contribution to the estimation of population mean kinship. Twenty parents contributed to the sampled offspring, five and two of which were dams and sires, respectively, that were born in the reserve. No mating between parent-offspring, or any of the aunt-uncle-nephew-niece pairs or first cousins was suggested by the assignments. However, we found that one offspring with ID 146 was produced by a half-sib parental pair (IDs 124 and 131). The individuals considered to be candidates for translocation were the bulls with IDs 176, 156, 320, 167, 144, 111, 145 and 271.

\section{Population demographic parameters}

Reproductive and demographic parameters of the population are shown in Table 3. The mean age at first calving among dams born in the reserve was 6.8 years, while two sires that were born in the reserve sired their first offspring at the age of 10 and 14 years. Multiparous dams had an average $C I$ of 3.7 years. The mean total number of offspring produced between 1993 and 2013 was 5.8 per sire and 2.4 per dam. The total of 45 sampled offspring out of 48 that were born in the reserve during the period of this study contributed to $15.9 \%$ and $18.6 \%$ of mean $H G$ and $A C R$, respectively. There was no deviation from a 50:50 natal sex ratio, with 22 males and 23 females.

Four bulls (ID 999, 130, 124, and 120) sired more than $50 \%$ of offspring for at least 1 year; however, only two bulls (999 and 130) showed the expected pattern of reproductive dominance since they successively showed exclusive paternity for consecutive years between 1996 and 2005 (Fig. 3). Following the translocations of 130 in 2005, four competing bulls (ID 120, 124, 133, and 170) sired offspring born between 2006 and 2012 .

\section{Discussion}

Our study demonstrates the value of combining genetic information with field observations to construct pedigrees to estimate relatedness and infer population demographic parameters, even when markers are not variable enough to produce distinct multilocus genotypes for every individual. Moreover, we found that management practices that include ear notching for individual identification significantly improved the field-observed assignments, particularly when combined with exclusion of incompatible molecular marker combinations. We could not find previous studies that quantified the effect of ear-notching and close observation on maternity assignment, but this is an encouraging finding. Importantly, we also found that multiple subordinate bulls were able to reproduce simultaneously, when formerly dominant bulls were removed. This has important implications for management practices, since it has been assumed that a single behaviourally dominant bull contributes to offspring born in a population of SWRs (Owen-Smith 1977; Rachlow et al. 1998).

\section{The efficiency of parentage assignment based on combining approaches}

Pedigrees obtained from observational data of wild populations are commonly compromised by inaccuracy and incompleteness of the observations (Bérénos et al. 2014). Similarly, pedigrees of wild animals inferred from molecular data can suffer from low statistical power of the molecular markers (Wang 2007) and missing data due to incomplete sample collection (Pemberton 2008). In this study, we combined incomplete observational and molecular data to maximise the rate of successful assignments. Using observational records alone could unambiguously assign parental pairs to only $11 \%$ of the total offspring; while the incorporation of genotypic exclusion and the combination of different parentage assignment approaches increased the assignment rate to $27 \%$ and $64 \%$, respectively. This is despite the presence of only $2-4$ alleles per microsatellite loci used. These results suggest that, even for populations with low genetic diversity, the combination of observational records and molecular markers could significantly improve the population pedigree regarding the proportion of unambiguous parental assignments. The rates of successful parentage assignment obtained in this study were relatively lower than previous SWR studies. Guerier et al. (2012) constructed a complete pedigree of a managed SWR population of 31 individuals by achieving $100 \%$ assignment rate of both parents for all 23 offspring using a combination of 11 microsatellite loci sampled from 


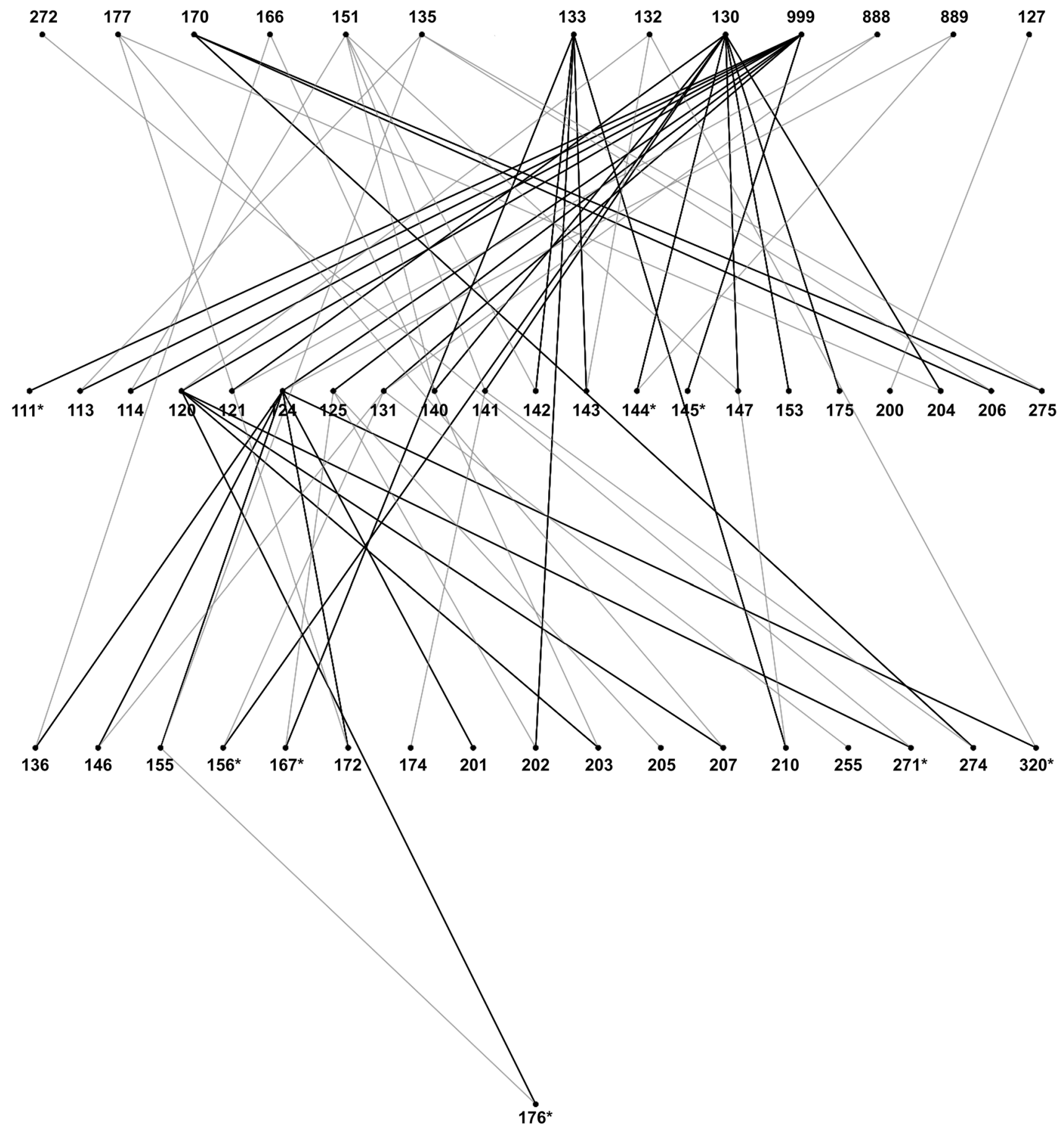

Fig. 2 Pedigree D drawn using PEDANTICS, illustrating all assignable parent-offspring pairs. Black and grey lines indicate paternity and maternity relationships, respectively. Asterisks indicate candi- dates suggested for translocation based on mean kinship. Note that the unsampled founders are individuals 999, 888, 889, and 127

paternity assignment could be obtained with confidence for only six offspring. The results of these studies suggested that smaller number of candidate parents and the capacity to sample all individuals in the populations were the important factors to achieve a high assignment rate. Although the number of markers used in parentage 
Table 3 Demographic information of the population determined using the final pedigree, showing the predicted values and their standard deviations, along with the number of observations used in estimation of the parameters $(\mathrm{N})$

\begin{tabular}{|c|c|c|c|}
\hline Parameter & Value & S.D. & $\mathrm{N}$ \\
\hline Mean dam age at first calving & 6.8 & 1.0 & 5 dams born in reserve \\
\hline Mean total no. of calves per sire & 5.8 & 2.1 & 6 reproductive bulls \\
\hline Mean total no. of calves per dam & 2.4 & 1.2 & 14 reproductive dams \\
\hline Mean calving interval (years) $(\mathrm{CI})$ & 3.7 & 1.7 & 10 multiparous dams, 19 intervals \\
\hline Natal sex ratio (male:female) ${ }^{\mathrm{a}}$ & $22: 23$ & - & 45 offspring \\
\hline Mean percentage of herd growth (HG) & 15.9 & 13.7 & 21 years \\
\hline Mean annual calving rate (ACR) & 18.6 & 15.1 & 21 years \\
\hline Effective population size $\left(N_{e}\right)^{\mathrm{b}}$ & 20 & - & - \\
\hline
\end{tabular}

${ }^{a}$ Deviation from an expected 50:50 sex ratio

${ }^{\mathrm{b}}$ Estimated using the number of breeding individual

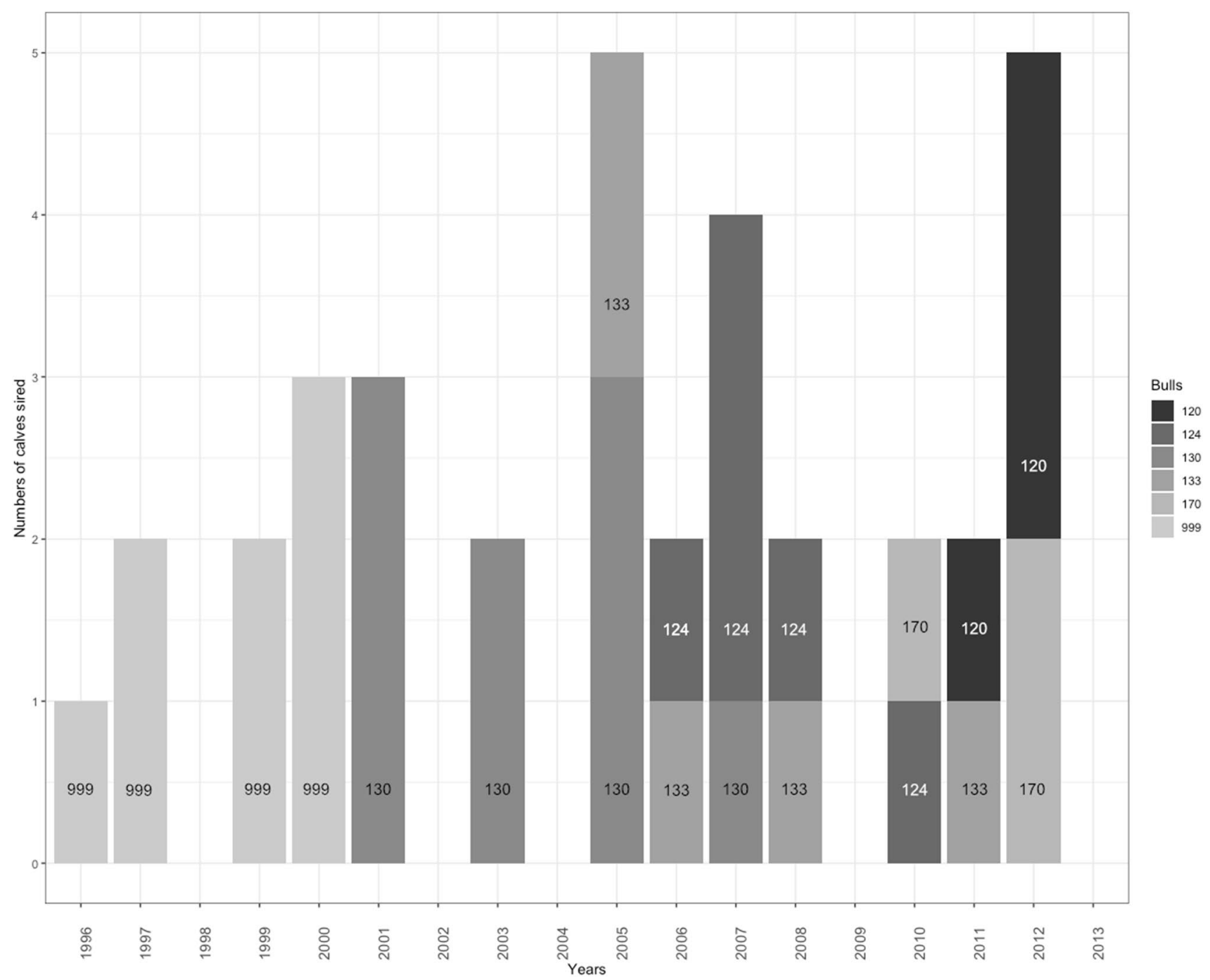

Fig. 3 The number of calves sired by six breeding bulls (grey scale and ID numbers represent the contribution of different bulls) reflecting the pattern of reproductive dominance in the population. The original dominant bulls have been translocated to other reserves: individual 999 in 1999; 130 in 2005 and 120 and 124 in 2012 
assignment has also been recognised as another succeeding factor for parentage assignment (Pemberton 2008); the study of Guerier et al. (2012) demonstrated that, even with low $N_{a}$, the complete assignment could be obtained by incorporating a well-maintained observation record. However, given the level of genetic diversity of the species, the number of currently available markers, and the capacity to maintain observational records with high accuracy; either observational-based or molecular-based assignment alone is insufficient to obtain a complete pedigree. One possible means of obtaining a complete pedigree would be to include more genetic markers in the parentage assignments, which recently has become more feasible because the introduction of next-generation sequencing allows the discovery of thousands of markers for non-model species. However, even with a larger set of markers, DNA samples from all animals in a population are still necessary to minimise erroneous assignment even though incomplete sampling can be taken into account in many parentage assignment softwares (Kalinowski et al. 2007; Walling et al. 2010). Here we further demonstrated the improvement of field-observed parentage assignments following the introduction of ear-notching; however, the overlap of 3 years between the starting of ear-notching (2006) and the cutoff year (2008) might lead to an upward bias in the assignment rate of the former period, as some animals were already marked. Regarding the construction of Pedigree $\mathrm{C}$, the Bayesian approach identified many fewer parentage assignments than CERVUS (only 5/76 pairs considered, compared to 23/33 trios considered, respectively). When we deliberately increased the critical value of the posterior probability in Bayesian-based assignments from 0.05 to 0.2 , this resulted in an increase of assignable parent-offspring pairs to $13 / 76$. Ten of these pairs were still consistent with the assignments made by likelihoodbased assignments. So, even with a more relaxed threshold for SOLOMON, CERVUS was more informative for this dataset. CERVUS takes the genotypes of the second most likely animal into consideration whereas SOLOMON determines confidence levels based on the expected probability of false parent-offspring pairs simulated from genotypes of the population (Walling et al. 2010). The outperformance of CERVUS may suggest that the likelihood-based approach is less sensitive to the low genetic variation in our data.

\section{Population genetic parameters}

Based on the genetic parameters determined in this study, we found that the level of genetic diversity was not as low as might be expected from the historical bottleneck. Means of parameters that described population heterozygosity such as $H_{o}, H_{e}$, and $F_{i s}$ in this study were similar to those found in other SWR genetic studies (Coutts 2009; Guerier et al. 2012; Harper et al. 2013; Labuschagne et al. 2017). The mean $H_{o}$ estimated from microsatellite markers in other SWR studies ranged from 0.440 to 0.478 ; while average $H_{o}$ found in this study was 0.426 . Lower heterozygosities have been reported in other mammal species that also experienced historical bottlenecks (Pertoldi et al. 2010; Corti et al. 2011; Fitak 2014). We did not find evidence of inbreeding but instead found a significant global excess of heterozygotes across loci. The negative mean value of $F_{i s}$ across loci also indicated that individuals in the population were less related than we would expect, although this was not significantly different from zero. The effects of metapopulation management by mixing previously separated populations could be responsible for the low inbreeding level found in this study. For example, observed heterozygosity was shown to be higher for F0 and F1 animals than for F2 animals in a managed SWR population in Namibia for which F0 animals were translocated from different locations (Guerier et al. 2012). Despite the fact that no evidence for inbreeding was found in this study, gradual loss of heterozygosity is likely to occur in managed populations of SWRs that implement similar conservation strategies. Thus, monitoring of genetic diversity in subsequent generations is still necessary to prevent inbreeding. In addition to heterozygosity, the number of alleles per locus is not only an indicator for measuring genetic variation but it is also an important factor to achieve high parentage assignment rate (Bernatchez and Duchesne 2000). The $N_{a}$ of 2.5 in this study was severely low and could have hindered successful parentage assignment. This warrants the effort of developing a larger set of markers to compensate for the low $N_{a}$, polymorphisms of markers, and confidence level of assignment in SWR populations.

\section{Using mean kinship to identify individuals for translocation}

Translocation of individuals among populations has been demonstrated to reduce inbreeding coefficients and increase genetic variation in a range of endangered populations (Bouzat et al. 2009; Mickelberg 2011; Moraes et al. 2017). The family of F-statistics have been used to monitor inbreeding levels and to consider sources of introduced animals; however, they are likely to preserve the gene pool of the most genetically distinct population (Eding and Meuwissen 2001). In contrast, using mean kinship at the individual level tends to preserve the gene pool of a founder population and is less sensitive to levels of genetic diversity (Jost 2008); hence, monitoring of mean kinship would provide the means to maintain a current level of genetic diversity (Willoughby et al. 2017) and prevent negative effects of fitness traits (Lacy et al. 2018). Further, mean kinship can also be estimated at a population level from molecular markers (Wang 
2011) and has been used to identify donor populations that contain valuable genetic resources in a variety of fragmented species (Finger et al. 2011; Mickelberg 2011; Garbe et al. 2016; Frankham et al. 2017). Outbreeding depression could be expected after translocation made between subpopulations with no recent geneflow (Frankham et al. 2017). To prevent the harmful effects of outbreeding, genetic status of populations of interest should be carefully studied and taken into consideration in any genetic rescue programme. However, we argue that outbreeding depression is unlikely the case for SWR as most modern populations originated from the same founder population just a couple of generations ago. Here we identified eight non-breeding bulls as candidates for translocation based on their high mean kinship. These bulls would be expected to provide a high risk of inbreeding in the population if they were retained and were to become reproductively active. Five out of the eight candidates already have been relocated to other reserves, two (ID 176 and 156) have been killed by other bulls, and only one candidate (ID 271) currently remains at the reserve. Of the six reproductive bulls (Fig. 3), all four of the dominant bulls had already been relocated (one in 1999, one in 2005 and two in 2012), leaving two bulls that have left offspring on the reserve. The proposal for male rather than female translocations (or other means of removal, including culling) is based on evidence that the prevalence of fighting and possibly killing other animals is increased when a higher number of adult males are kept within a particular area $(\mathrm{Du}$ Toit 2006). Moreover, translocation of males is a more costefficient strategy for introducing new diversity into a population than moving females, because males are likely to have a more substantial genetic contribution, as demonstrated by the average number of offspring per bull and dam observed in this study. We suggest that translocation of young bulls with high kinship together with regular relocating of dominant bulls could retard the rate of inbreeding in SWR populations. The determination of a justifiable upper limit to population mean kinship that should be maintained in wild populations is difficult. Frankham et al. (2017) suggested using the value of 0.1 as a practical guideline. Although the population mean kinship of 0.0483 reported in this study was substantially lower than the suggested level, the value was likely to be underestimated, based on the assumption that founder animals were unrelated.

\section{Male dominance}

Our study also demonstrated the value of pedigrees for assessing reproductive success rather than relying on observed behaviour to infer the social structure of animals with suspected dominance hierarchies. A dominant bull solitarily occupies a territory that may be shared with other subordinate bulls that are normally constrained within a single territory (Owen-Smith 1975; Thompson et al. 2016). When a dominant bull encounters a cow in estrous, the cow is confined within the territory until successfully mated (Kingdon and Hoffmann 2013). Therefore, a dominant bull is expected to spend more time with reproductive females (Rachlow et al. 1998) and to be more reproductively successful than sub-ordinate bulls (Owen-Smith 1977). However, in this study, we found that the reproductive dominance of bulls was not as complete as expected following the removal of two dominant founder bulls. Sub-territories within the reserve might have developed as a result of the population growing and following the construction of a new waterhole in the reserve that would allow multiple nonoverlapping territories of the bulls. There are few genetic studies that have successfully obtained paternity assignment in SWR populations that would allow researchers to determine the dominant reproductive behaviour of the species because most studies have been unable to assign the paternity of offspring with statistical confidence (Coutts 2009; Labuschagne et al. 2017). However, one study conducted in a limited free-ranging population that held two founder bulls reported that the bull that was believed to be subordinate had actually sired 10 of 13 offspring during a 9-year period. Additionally, two newly introduced bulls had succeeded in breeding before they established their territories (Guerier et al. 2012). Our finding and that of Guerier et al. (2012) contradict the hypothesis that only a single behaviourally dominant bull contributes to the offspring born in a population. With more contributing bulls presented in our study, the results provided clearer insight into the breeding pattern in limited free-ranging populations. In regard to multiple contributing bulls, this also provided evidence to support the hypothesis that regular translocations of dominant bulls could encourage sub-ordinate bulls to breed; hence, slowing rates of inbreeding.

\section{Population demographic parameters}

The final pedigree was useful for estimating other population demographic parameters that could influence the effectiveness of intervention strategies. No evidence of natal sex-ratio skewness was found, which supports previous studies. Natal sex-ratio skewness toward males has been reported in captive SWRs (Zschokke et al. 1998; Kretzschmar 2001; Linklater 2007), whereas non-captive populations kept at lower stocking densities have been reported to be in the expected 50:50 sex ratio (Ververs et al. 2017). The average ACR and $C I$ observed in this study were $18.6 \%$ and 3.7 years, respectively; which could be interpreted as a very poor fecundity population based on the guidelines provided by the Southern African Development Community Rhino Management Group (Du Toit 2006). The reproductive performance of the population in this study was less than that reported in other 
non-captive populations, for which $A C R$ and $C I$ ranged from 20 to $50 \%$ and 2.4 to 3.3 years, respectively (Rachlow and Berger 1998; Kretzschmar 2001; Ververs et al. 2017). The ACR target is a value greater than $33 \%$, which corresponds to a $C I$ of 3 years (Du Toit 2006). The high variability of observed $A C R$ in this study, which ranged from 0 to $50 \%$, resulted from a small number of breeding dams and was the main reason for the low apparent fecundity of the population in this study. Aberrant ovarian cycles and pathological lesions of female reproductive tracts have been reported in SWRs kept in both captivity and non-captivity, and these defects are believed to cause low reproductive performance (Hermes et al. 2006; Roth et al. 2018; Ververs 2018). Studies conducted in captive populations led to the hypothesis that prolonged estrogenic exposure from phytoestrogens found in plants that the cows fed on may be responsible for the aberrant ovarian function (Tubbs et al. 2017). Although the accessibility of non-captive SWRs to phytoestrogen-rich plants is limited, it may be worth investigating whether such plant species exist in the habitats of populations with poor fecundity. Another possible explanation for low fecundity is the effect of inbreeding as a consequence of a historical population bottleneck; however, it may be difficult or even impossible to confirm the effects of inbreeding with sufficient statistical confidence in real SWR populations. Krummenacher and Zschokke (2007) found a slight, but not significant, increase in mortality rate of inbred SWRs using data from international studbook records. However, a power analysis indicated that they would have needed more than 640 inbred offspring born in the international zoos to detect a statistical difference given the reported mortality rate. For the estimation of $C I$, we additionally estimated the adjusted $C I$, which accounted for the unassignable offspring and three offspring that died before being sampled. However, the adjusted $C I$ of 2.0 was not consistent with the ACR that was estimated from all 45 offspring and indicated low fecundity of the population. The unequal contribution of females could be responsible for this inconsistency; therefore, using $\mathrm{CI}$ estimated only from the 33 assignable dam-offspring pairs was more reasonable to represent the reproductive success of the population. To estimate mean kinship of a population with incomplete pedigree, either using of average mean kinship or assuming no contribution for a missing relationship has been used (Cassell et al. 2003). Here, we assumed no input from the missing data. This approach would limit the extent of overestimated mean kinship, while the extent of underestimated mean kinship could be substantial given the historical genetic bottleneck of the species.

\section{Conclusions}

Conservation strategies that aim to protect SWRs within secured areas has contributed to an increase in the total number of SWR, but have generated small and fragmented populations, raising concerns about the genetic viability of the species. Translocation has been implemented as a tool to increase gene flow among populations to maintain the current level of genetic diversity of the species. Here we have demonstrated that records of field-observations with a high degree of ambiguity could be improved by the incorporation of genetic markers, even for populations with low levels of diversity. The pedigree thus allowed us to use the kinship coefficient to quantitatively identify the best candidates for translocations to maintain the current genetic diversity of the population. Indications from the pedigree that multiple bulls contributed to a group of offspring born in the same year is valuable information and can be used in genetic management of SWR populations. Although we have demonstrated that using mean kinship to monitor level of inbreeding provided a tool to incorporate genotypes and observations records for metapopulation management, a larger set of markers is necessary to maximise the assignment rate.

Acknowledgements We wish to thank Dr. Cindy Harper of the Onderstepoort Veterinary Genetics Laboratory for the microsatellite genotyping and the comments of two anonymous reviewers that substantially improved the manuscript. Our special thanks are extended to the Department of Wildlife and National Parks of Botswana for the collaboration and we hope that this study will be useful for the further decision making in the conservation of the southern white rhinoceros.

Open Access This article is distributed under the terms of the Creative Commons Attribution 4.0 International License (http://creativeco mmons.org/licenses/by/4.0/), which permits unrestricted use, distribution, and reproduction in any medium, provided you give appropriate credit to the original author(s) and the source, provide a link to the Creative Commons license, and indicate if changes were made.

\section{References}

Agresti A (1992) A survey of exact inference for contingency tables. Stat Sci 7:131-153. https://doi.org/10.1214/ss/1177011454

Ballou JD, Lacy RC (1995) Identifying genetically important individuals for management of genetic variation in pedigreed populations. In: Ballou J, Gilpin M, Foose T (eds) Population management for survival and recovery. Columbia University Press, New York, $p$ 76-111

Bérénos C, Ellis PA, Pilkington JG, Pemberton JM (2014) Estimating quantitative genetic parameters in wild populations: a comparison of pedigree and genomic approaches. Mol Ecol 23:3434-3451. https://doi.org/10.1111/mec.12827

Bernatchez L, Duchesne P (2000) Individual-based genotype analysis in studies of parentage and population assignment: how many loci, how many alleles? Can J Fish Aquat Sci 57:1-12. https:// doi.org/10.1139/f99-271 
Bouzat JL, Johnson JA, Toepfer JE, Simpson SA, Esker TL, Westemeier RL (2009) Beyond the beneficial effects of translocations as an effective tool for the genetic restoration of isolated populations. Conserv Genet 10:191-201. https://doi.org/10.1007/s1059 2-018-1098-Z

Cassell BG, Adamec V, Pearson RE (2003) Effect of incomplete pedigrees on estimates of inbreeding and inbreeding depression for days to first service and summit milk yield in holsteins and jerseys. J Dairy Sci 86:2967-2976. https://doi.org/10.3168/jds.S0022 $-0302(03) 73894-6$

Christie MR, Tennessen JA, Blouin MS (2013) Bayesian parentage analysis with systematic accountability of genotyping error, missing data and false matching. Bioinformatics 29(6):725-732. https ://doi.org/10.1093/bioinformatics/btt039

Corti P, Shafer ABA, Coltman DW et al (2011) Past bottlenecks and current population fragmentation of endangered huemul deer (Hippocamelus bisulcus): implications for preservation of genetic diversity. Conserv Genet 12:119. https://doi.org/10.1007/s1059 2-009-9997-7

Coutts NJ (2009) Investigating genetic diversity at neutral and adaptive DNA markers in the severly bottlenecked southern white rhinoceros (Ceratotherium simum simum). Dissertation, University of Cape Town

Department of Wildlife and National Parks of Botswana (2002) Conservation and management strategy for the white rhinoceros Ceratotherium simum and the black rhinoceros Diceros bicornis in Botswana. Department of Wildlife and National Parks Botswana, Gaborone

Du Toit RF (2006) Guidelines for implementing SADC rhino conservation strategies. WWF-SARPO, Harare

Eding H, Meuwissen THE (2001) Marker-based estimates of between and within population kinships for the conservation of genetic diversity. J Anim Breed Genet 118:141-159. https://doi.org/10.1 046/j.1439-0388.2001.00290.x

Emslie R, Brooks M (1999) African rhino: status survey and conservation action plan. https://www.iucn.org/content/african-rhino-statu s-survey-and-conservation-action-plan. Accessed 4 Jan 2018

Emslie R, Amin R, Kock R (2009) Guidelines for the in situ re-introduction and translocation of African and Asian rhinoceros. https ://www.iucn.org/content/guidelines-situ-re-introduction-and-trans location-african-and-asian-rhinoceros. Accessed 4 Jan 2018

Emslie RH, Milliken T, Talukdar B, Ellis S, Adcock K, Knight MH (2016) African and Asian rhinoceroses-status, conservation and trade. In: A report from the IUCN Species Survival Commission (IUCN/SSC) African and Asian Rhino Specialist Groups and TRAFFIC to the CITES Secretariat pursuant to Resolution Conf. CoP17 Doc. 68 Annex 5, Johannesburg, South Africa

Falconer DS (1960) Introduction to quantitative genetics. Oliver and Boyd, Edinburgh

Finger A, Kettle CJ, Kaiser-bunbury CN, Valentin T, Doudee D, Matatiken D, Ghazoul J (2011) Back from the brink: potential for genetic rescue in a critically endangered tree. Mol Ecol 20:37733784. https://doi.org/10.1111/j.1365-294X.2011.05228.x

Fitak RR (2014) Conservation genomics of the endangered Mexican wolf and de novo SNP marker development in pumas using nextgeneration sequencing. Dissertation, University of Arizona

Florescu A et al (2003) Polymorphic microsatellites in white rhinoceros. Mol Ecol Notes 3:344-345. https://doi.org/10.104 6/j.1471-8286.2003.00440.x

Frankham R et al (2017) Genetic management of fragmented animal and plant populations. Oxford University Press, Oxford

Garbe JR, Prakapenka D, Tan C, Da Y (2016) Genomic inbreeding and relatedness in wild panda populations. PLoS ONE 11(8):e0160496. https://doi.org/10.1371/journal.pone.0160496
Guerier AS, Bishop JM, Crawford SJ, Schmidt-Küntzel A, Stratford KJ (2012) Parentage analysis in a managed free ranging population of southern white rhinoceros: genetic diversity, pedigrees and management. Conserv Genet 13:811-822. https://doi.org/10.1007/ s10592-012-0331-4

Harper CK, Vermeulen GJ, Clarke AB, Jacobus I, Guthrie AJ (2013) Extraction of nuclear DNA from rhinoceros horn and characterization of DNA profiling systems for white (Ceratotherium simum) and black (Diceros bicornis) rhinoceros. Forensic Sci Int Genet 7:428-433. https://doi.org/10.1016/j.fsigen.2013.04.003

Hermes R et al (2006) The effect of long non-reproductive periods on the genital health in captive female white rhinoceroses (Ceratotherium simum simum, C.s. cottoni). Theriogenology 65:1492-1515. https://doi.org/10.1016/j.theriogenology.2005.09.002

Jost LOU (2008) $\mathrm{G}_{\mathrm{ST}}$ and its relatives do not measure differentiation. Mol Ecol 17:4015-4026. https://doi.org/10.1111/j.1365294X.2008.03887.x

Kalinowski ST, Taper ML, Marshall TC (2007) Revising how the computer program CERVUS accommodates genotyping error increases success in paternity assignment. Mol Ecol 16:10991106. https://doi.org/10.1111/j.1365-294X.2007.03089.x

Kingdon J, Hoffmann M (2013) Mammals of Africa. Volume V: carnivores, pangolins, equids and rhinoceroses. Bloomsbury Publishing, London

Kretzschmar P (2001) Population growth, sex ratio and reproduction of a natural living population of white rhinoceros. In: Recent research on elephants and rhinos, abstracts of the international elephant and rhino research symposium. Zoologischer Garten, Vienna, Austria. pp 196-201

Krummenacher TS, Zschokke S (2007) Inbreeding and outbreeding in African rhinoceros species. Pachyderm 42:108-115

Labuschagne C, Dalton DL, Grobler JP, Kotzé A (2017) SNP discovery and characterisation in white rhino (Ceratotherium simum) with application to parentage assignment. Genet Mol Biol 40:84-92. https://doi.org/10.1590/1678-4685-GMB-2016-0058

Lacy RC, Malo AF, Alaks G (2018) Maintenance of genetic variation in quantitative traits of a woodland rodent during generations of captive breeding. Conserv Genet 19:789-802. https://doi. org/10.1007/s10592-018-1054-y

Linklater WL (2007) Translocation reverses birth sex ratio bias depending on its timing during gestation: evidence for the action of two sex-allocation mechanisms. Reprod Fertil Dev 19:831-839. https ://doi.org/10.1071/RD07027

Marshall T, Slate J, Kruuk L, Pemberton J (1998) Statistical confidence for likelihood-based paternity inference in natural populations. Mol Ecol 7:639-655. https://doi.org/10.1046/j.1365294x.1998.00374.x

Mickelberg JL (2011) Understanding and managing isolation in a fragmented population of golden lion tamarins, Leontopithecus rosalia. Dissertation, George Mason University

Moraes AM et al (2017) Temporal genetic dynamics of reintroduced and translocated populations of the endangered golden lion tamarin (Leontopithecus rosalia). Conserv Genet 18:995-1009. https ://doi.org/10.1007/s10592-017-0948-4

Morrissey MB, Wilson AJ (2010) Pedantics: an R package for pedigree-based genetic simulation and pedigree manipulation, characterization and viewing. Mol Ecol Resour 10(4):711-719. https ://doi.org/10.1111/j.1755-0998.2009.02817.x

Owen-Smith RN (1975) The social ethology of the white rhinoceros Ceratotherium simum (Burchell 1817). Ethol 38:337-384. https ://doi.org/10.1111/j.14390310.1975.tb02010.x

Owen-Smith N (1977) On territoriality in ungulates and an evolutionary model. Q Rev Biol 52:1-38. https://doi.org/10.1086/409720

Pemberton JM (2008) Wild pedigrees: the way forward. Proc R Soc B Biol Sci 275:613-621. https://doi.org/10.1098/rspb.2007.1531 
Pertoldi C et al (2010) Genome variability in European and American bison detected using the BovineSNP50 BeadChip. Conserv Genet 11:627-634. https://doi.org/10.1007/s10592-009-9977-y

Player I (1967) Translocation of white rhinoceros in South Africa. Oryx 9:137-150. https://doi.org/10.1017/S0030605300006165

Rachlow JL, Berger J (1998) Reproduction and population density: trade-offs for the conservation of rhinos in situ. Anim Conserv 1:101-106. https://doi.org/10.1111/j.1469-1795.1998.tb00017.x

Rachlow JL, Berkeley EV, Berger J (1998) Correlates of male mating strategies in white rhinos (Ceratotherium simum). J Mammal 79:1317-1324. https://doi.org/10.2307/1383023

Raymond M, Rousset F (1995) An exact test for population differentiation. Evolution 49:1280-1283. https://doi. org/10.1111/j.1558-5646.1995.tb04456.x

Roth TL, Schook MW, Stoops MA (2018) Monitoring and controlling ovarian function in the rhinoceros. Theriogenology 109:48-57. https://doi.org/10.1016/j.theriogenology.2017.12.007

Sambrook J, Russell DW (2006) Purification of nucleic acids by extraction with phenol: chloroform. Cold Spring Harb Protoc. https:// doi.org/10.1101/pdb.prot 4455

Scott CA (2008) Microsatellite variability in four contemporary rhinoceros species: implications for conservation. Dissertation, Queen's University

Sinnwell JP, Therneau TM, Schaid DJ (2014) The Kinship2 R package for pedigree data. Hum Hered 78:91-93. https://doi. org/10.1159/000363105

Thompson S, Avent T, Doughty LS (2016) Range analysis and terrain preference of adult southern white rhinoceros (Ceratotherium simum) in a South African private game reserve: insights into carrying capacity and future management. PLoS ONE 11:e0161724. https://doi.org/10.1371/journal.pone.0161724

Tjibae M (2001) SADC Regional Programme for Rhino Conservation. In: SADC Rhino Range States and Consortium Meeting, Pilanesberg, pp 4-5

Tubbs CW, Durrant BS, Milnes MR (2017) Reconsidering the use of soy and alfalfa in southern white rhinoceros diets. Pachyderm $58: 135-139$
Verreynne FJ (2012) Update of the central database and studbook for all southern white rhinoceros (Cerathotherium simum simum) under private management in the Republic of Botswana. Dissertation, University of Pretoria

Ververs C (2018) Breeding on the brink of extinction: what can we learn from game-ranched white rhinoceros (Ceratotherium simum simum). Dissertation, Ghent University

Ververs C, van Zijll Langhout M, Hostens M, Otto M, Govaere J, Durrant B, Van Soom A (2017) Reproductive performance parameters in a large population of game-ranched white rhinoceroses (Ceratotherium simum simum). PLoS ONE 12:e0187751. https://doi. org/10.1371/journal.pone.0187751

Walling CA, Pemberton JM, Hadfield JD, Kruuk LE (2010) Comparing parentage inference software: reanalysis of a red deer pedigree. Mol Ecol 19(9):1914-1928. https://doi.org/10.1111/j.1365294X.2010.04604.X

Wang J (2007) Parentage and sibship exclusions: higher statistical power with more family members. Heredity 99(2):205-217. https ://doi.org/10.1038/sj.hdy.6800984

Wang J (2011) COANCESTRY: a program for simulating, estimating and analysing relatedness and inbreeding coefficients. Mol Ecol Resour 11:141-145. https://doi.org/10.111 1/j.1755-0998.2010.02885.x

Willoughby JR, Ivy JA, Lacy RC, Doyle JM, DeWoody JA (2017) Inbreeding and selection shape genomic diversity in captive populations: implications for the conservation of endangered species. PLoS ONE 12:e0175996. https://doi.org/10.1371/journ al.pone. 0175996

Zschokke S, Studer P, Baur B (1998) Past and future breeding of the Indian rhinoceros in captivity. Int Zoo News 5:261-276. https:// conservation.unibas.ch/team/zschokke/pdf/zschokke1998izn.pdf

Publisher's Note Springer Nature remains neutral with regard to jurisdictional claims in published maps and institutional affiliations. 